\title{
Efficient Sequential and Parallel Algorithms for Estimating Higher Order Spectra
}

\author{
Zigeng Wang \\ Dept. of Computer Science \& Engr. \\ University of Connecticut \\ Storrs, Connecticut, U.S.A \\ zigeng.wang@uconn.edu
}

\author{
Abdullah-Al Mamun* \\ Dept. of Computer Science \& Engr. \\ University of Connecticut \\ Storrs, Connecticut, U.S.A \\ abdullah.am.cs@gmail.com
}

\author{
Xingyu Cai \\ Dept. of Computer Science \& Engr. \\ University of Connecticut \\ Storrs, Connecticut, U.S.A \\ xingyu.cai@uconn.edu
}

\author{
Nalini Ravishanker \\ Dept. of Statistics \\ University of Connecticut \\ Storrs, Connecticut, U.S.A \\ nalini.ravishanker@uconn.edu
}

\begin{abstract}
Higher order spectra (HOS) are a powerful tool in nonlinear time series analysis and they have been extensively used as feature representations in data mining, communications and cosmology domains. However, HOS estimation suffers from high computational cost and memory consumption. Any algorithm for computing the $k$ th order spectra on a dataset of size $n$ needs $\Omega\left(n^{k-1}\right)$ time since the output size will be $\Omega\left(n^{k-1}\right)$ as well, which makes the direct HOS analysis difficult for long time series, and further prohibits its direct deployment to resource-limited and time-sensitive applications. Existing algorithms for computing HOS are either inefficient or have been implemented on obsolete architectures. Thus it is essential to develop efficient generic algorithms for HOS estimations.

In this paper, we present a package of generic sequential and parallel algorithms for computationally and memory efficient HOS estimations which can be employed on any parallel machine or platform. Our proposed algorithms largely reduce the HOS' computational cost and memory usage in spectrum multiplication and smoothing steps through carefully designed prefix sum operations. Moreover, we employ a matrix partitioning technique and design algorithms with optimal memory usage and present the parallel approaches on the PRAM and the mesh models. Furthermore, we implement our algorithms for both bispectrum and trispectrum estimations. We conduct extensive experiments and cross-compare the proposed algorithms' performance. Results show that our algorithms achieve state-of-the-art computational and memory efficiency, and our parallel algorithms achieve close to linear speedups. The code is available at https://github.com/ZigengWang/HOS.
\end{abstract}

\footnotetext{
*Abdullah-Al is currently affliated with Google LLC, Mountain View, California, U.S.A.

${ }^{\dagger}$ Sanguthevar Rajasekaran is the corresponding author.

Permission to make digital or hard copies of all or part of this work for personal or classroom use is granted without fee provided that copies are not made or distributed for profit or commercial advantage and that copies bear this notice and the full citation on the first page. Copyrights for components of this work owned by others than ACM must be honored. Abstracting with credit is permitted. To copy otherwise, or republish, to post on servers or to redistribute to lists, requires prior specific permission and/or a fee. Request permissions from permissions@acm.org.

CIKM '19, November 3-7, 2019, Beijing, China

(C) 2019 Association for Computing Machinery.

ACM ISBN 978-1-4503-6976-3/19/11 ..\$15.00

https://doi.org/10.1145/3357384.3358062
}

\author{
Sanguthevar Rajasekaran ${ }^{\dagger}$ \\ Dept. of Computer Science \& Engr. \\ University of Connecticut \\ Storrs, Connecticut, U.S.A \\ sanguthevar.rajasekaran@uconn.edu
}

\section{CCS CONCEPTS}

- Mathematics of computing $\rightarrow$ Time series analysis; • Theory of computation $\rightarrow$ Shared memory algorithms.

\section{KEYWORDS}

higher order spectra; sequential algorithms; parallel algorithms; memory efficient algorithms; linear speedups

\section{ACM Reference Format:}

Zigeng Wang, Abdullah-Al Mamun, Xingyu Cai, Nalini Ravishanker, and Sanguthevar Rajasekaran. 2019. Efficient Sequential and Parallel Algorithms for Estimating Higher Order Spectra. In The 28th ACM International Conference on Information and Knowledge Management (CIKM'19), November 3-7, 2019, Beijing, China. ACM, New York, NY, USA, 10 pages. https: //doi.org/10.1145/3357384.3358062

\section{INTRODUCTION}

Spectral analysis is a fundamental domain in signal processing and time series data mining. Power spectrum, the Fourier transform of second order cumulants of a sequence, is a useful tool to analyze the autocorrelation of a stationary Gaussian process. However, in many practical applications, the information beyond the autocorrelation has to be captured, such as the nonlinear interactions in time series. Higher order spectra (HOS), the Fourier transform pair of the third (bispectrum, shown in Figure 1) or even higher order cumulants which contain such nonliear interactions, are receiving growing research interests in recent decades.
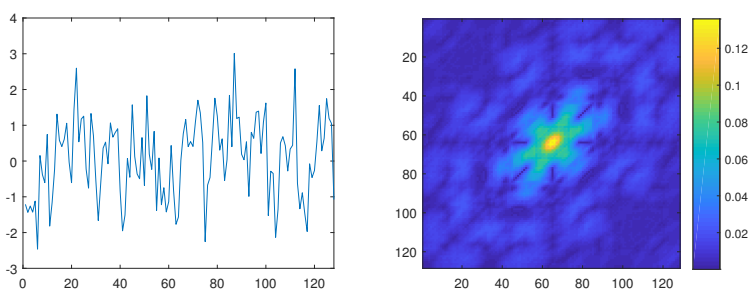

Figure 1: Time Series and its Bispectrum

Recently, in communications engineering, a bispectrum-based ultra wideband (UWB) interference-robust communication system was proposed [13]. In systems analysis, HOS have been used to characterize nonlinear systems with different input distributions [14]. 
In cosmology, a distinctive bispectrum of primordial perturbation from massive spinning particles is discussed to describe our universe during inflation [3]. More broadly, in the data mining and machine learning domains, the bispectrum has been extensively employed as a meaningful feature representation and further fed into modern learning models, which has shown promising results in seizure prediction [2], emotions recognition [21], chemical reaction estimation [5], and satellite-based precipitation [19].

HOS have a promising property of characterizing nonlinearities but also require expensive computations. HOS estimation has a exponential order dependency. For computing the $k$ th order spectra on a sequence of length $n$, it needs at least $\Omega\left(n^{k-1}\right)$ time. Taking the bispectrum as an example, as shown in Figure 1, it augments the original 1-D sequence of length 128 to 2-D matrix of size $128^{2}$. Thus, it is difficult to directly deploy HOS to time-sensitive or resource-limited devices, such as real-time UWB communication systems [13] or wearable devices for seizure prediction [2]. Existing algorithms for HOS computation are either nongeneric or have been implemented on obsolete architectures [9, 10, 12]. In this paper, we propose a package of generic efficient sequential and parallel HOS algorithms specialized for different application requirements:

- For time-sensitive applications, we propose ultra-fast generic sequential and parallel algorithms on the PRAM in Section 5.2, empowered by specialized prefix sum operation. We theoretically and experimentally prove that our parallel algorithm achieves a linear speedup.

- For resource-limited applications, we propose memory efficient algorithms in Section 5.3 with matrix partitioning trick. Experiment results show that an optimal memory usage has achieved without sacrificing computational efficiency.

In this paper, a major part is devoted to a discussion on the bispectrum estimation. In Section 7 and Section 8, we show that the proposed techniques can be easily extended to even higher HOS (e.g. trispectrum).

Contributions of this paper:

We propose a package of computationally and memory efficient sequential and parallel algorithms for bispectrum and trispectrum estimation. The parallel algorithms on PRAM are generic in nature and can be employed on any parallel machine or platform. Theoretical analysis and experiments show that our algorithms achieve state-of-the-art runtime and memory usage. Results also show our parallel algorithms achieve close to linear speedups.

In the following, Section 2 introduces the preliminaries and reviews related works. Sections 3 and 4 present general speedup approaches for sequential and parallel HOS computation. Section 5 introduces the fast and memory efficient algorithms. Section 6 explains the mesh implementation for the bispectrum estimation. Section 7 discusses the polyspectra estimation of higher orders than the bispectrum. Section 8 details our experiments and Section 9 concludes the paper.

\section{PRELIMINARY AND RELATED WORK}

Problem Statement: If $X(t)$ is a stationary random process $(t$ denoting discrete time), the moments of order $k$ are given by [15]:

$$
m_{k}^{x}\left(\tau_{1}, \tau_{2}, \ldots, \tau_{k-1}\right)=E\left[X(t) X\left(t+\tau_{1}\right) \cdots X\left(t+\tau_{k-1}\right)\right] .
$$

where $E[$.$] denotes expectation and \tau_{1}, \tau_{2}, \ldots, \tau_{k-1}$ denote time lags. The $k$ th order cumulants are the moments of order up to $k$. In this manner, the first order cumulants are defined as $c_{1}^{x}=m_{1}^{x}=E[X(t)]$, the second order cumulants are defined as $c_{2}^{x}\left(\tau_{1}\right)=m_{2}^{x}\left(\tau_{1}\right)-\left(m_{1}^{x}\right)^{2}$, and so on.

\subsection{Standard Algorithms for HOS}

In this section we summarize the known algorithms for computing HOS, specifically, the bispectrum. Two kinds of algorithms can be found in the literature: an indirect method and a direct method. We summarize them as follows ${ }^{1}$ :

2.1.1 Indirect Method. Let $X(1), X(2), \ldots, X(n)$ be the input time series. Steps of the InDirect BiSpectral (IDBS) algorithm are:

\section{Algorithm IDBS}

1) Partition the input into $K$ parts with $M$ samples in each. Let $X^{i}$ stand for the $i$ th part, for $1 \leq i \leq \frac{n}{M}$.

2) In each part, subtract the mean of that part from each element.

3) Estimate the third moments of each part $X^{i}$ as follows:

$$
m_{3}^{x^{i}}\left(\tau_{1}, \tau_{2}\right)=\frac{1}{M} \sum_{l=l_{1}}^{l_{2}} X^{i}(l) X^{i}\left(l+\tau_{1}\right) X^{i}\left(l+\tau_{2}\right)
$$

where $l_{1}=\max \left(0,-\tau_{1},-\tau_{2}\right), l_{2}=\min \left(M, M-\tau_{1}, M-\tau_{2}\right)$ and $1 \leq i \leq K$. The superscript $x^{i}$ of $m_{3}^{x^{i}}$ indicates the moments are calculated with respect to elements in part $X^{i}$.

4) Compute the average cumulant as:

$$
\hat{c}_{3}^{x}\left(\tau_{1}, \tau_{2}\right)=\frac{1}{K} \sum_{i=1}^{K} m_{3}^{x^{i}}\left(\tau_{1}, \tau_{2}\right) .
$$

5) The bispectrum can now be estimated as:

$$
\hat{C}_{3}^{x}\left(\omega_{1}, \omega_{2}\right)=\sum_{\tau_{1}=-L}^{L} \sum_{\tau_{2}=-L}^{L} \hat{c}_{3}^{x}\left(\tau_{1}, \tau_{2}\right) e^{-j\left(\omega_{1} \tau_{1}+\omega_{2} \tau_{2}\right)} w\left(\tau_{1}, \tau_{2}\right) .
$$

where $j=\sqrt{-1}, L<M$, and $w\left(\tau_{1}, \tau_{2}\right)$ is a two-dimensional window used to smooth out edge effects. For a description of appropriate windows that can be used above please see [15].

Time Complexity Analysis: Step 2 of the indirect method takes $O(n)$ time. In Step 3, for a given pair $\left(\omega_{1}, \omega_{2}\right)$ and $i$, computation of $m_{3}^{x^{i}}$ takes $O(M)$ time. Thus, Step 3 takes a total of $O\left(M^{3} K\right)=$ $O\left(M^{2} n\right)$ time. Step 4 takes a total of $O\left(M^{2} K\right)=O(M n)$ time. Finally, Step 5 takes a total of $O\left(M^{2} L^{2}\right)=O\left(M^{4}\right)$ time.

In summary, the run time of the indirect method is $O\left(M^{2} n+M^{4}\right)$.

2.1.2 Direct Method. Let $X(1), X(2), \ldots, X(n)$ be the input time series. The Direct BiSpectral (DBS) algorithm works as follows:

\section{Algorithm DBS}

1) Partition the input into $K$ parts with $M$ samples in each part. Let $X^{i}$ stand for the $i$ th part, for $1 \leq i \leq \frac{n}{M}$.

2) In each part subtract the mean of that part from each element.

3) Compute the Discrete Fourier Transform $F^{x^{i}}(k)$ for each part:

$$
\begin{aligned}
F^{x^{i}} & (k)=\sum_{u=0}^{M-1} X^{i}(u) e^{-j \frac{2 \pi}{M} u k} \\
& \quad \text { for } k=0,1, \ldots, M-1 ; i=1,2, \ldots, K
\end{aligned}
$$

\footnotetext{
${ }^{1}$ Detailed HOS computation summaries can be found in $[15,16]$
} 
4) Estimate the bispectrum of each part as:

$$
\begin{aligned}
C_{3}^{x^{i}}\left(k_{1}, k_{2}\right)= & \frac{1}{M} F^{x^{i}}\left(k_{1}\right) F^{x^{i}}\left(k_{2}\right) F^{x^{i}}\left(k_{1}+k_{2}\right), \\
& \text { for } i=1,2, \ldots, K .
\end{aligned}
$$

Since the bispectrum has cetain symmetries, it is sufficient to compute $C_{3}^{x^{i}}\left(k_{1}, k_{2}\right)$ only in the region: $0 \leq k_{2} \leq k_{1}, k_{1}+k_{2}<M / 2$.

5) This step performs some smoothing over a window of size $M_{3} \times M_{3}$. This is done to reduce the variance of the estimate.

$$
\widetilde{C}_{3}^{x^{i}}\left(k_{1}, k_{2}\right)=\frac{1}{M_{3}^{2}} \sum_{n_{1}=-M_{3} / 2}^{M_{3} / 2-1} \sum_{n_{2}=-M_{3} / 2}^{M_{3} / 2-1} C_{3}^{x^{i}}\left(k_{1}+n_{1}, k_{2}+n_{2}\right) \text {. }
$$

6) The third-order spectrum is obtained by averaging over all parts:

$$
\hat{C}_{3}^{x}\left(\omega_{1}, \omega_{2}\right)=\frac{1}{K} \sum_{i=1}^{K} \tilde{C}_{3}^{x^{i}}\left(\omega_{1}, \omega_{2}\right), \omega_{1}=\frac{2 \pi}{M} k_{1} \text { and } \omega_{2}=\frac{2 \pi}{M} k_{2} .
$$

Time Complexity Analysis: Step 2 in the direct method takes $O(n)$ time. Step 3 takes a total of $O(n \log M)$ time. Step 4 takes $O\left(M^{2}\right)$ time per part. Thus Step 4 takes a total of $O\left(K M^{2}\right)=O(M n)$. In Step 5 smoothing is done. For every point $\left(k_{1}, k_{2}\right)$, the smoothed value $\widetilde{C}_{3}^{x^{i}}\left(k_{1}, k_{2}\right)$ is computed as the average value of $C_{3}^{x^{i}}$ over a region of size $O\left(M_{3}^{2}\right)$. Thus each such computation takes $O\left(M_{3}^{2}\right)$ time. The total time taken in Step 5 is $O\left(M^{2} K M_{3}^{2}\right)=O\left(M n M_{3}^{2}\right)$.

In summary, the total run time of the direct method is $O\left(M n M_{3}^{2}\right)$.

\subsection{Related Parallel Algorithms for HOS}

Some parallel algorithms for the bispectrum have been published in the 1990's. (see e.g., $[9,10,12])$. To the best of our knowledge, no papers have been published since then on the parallel computation of HOS. We now summarize the known algorithms.

Manolakos, et al. [12] discussed HOS in signal processing. Followed by this, they employed the canonical mapping methodology (CMM) to derive parallel programs for bispectrum computation. The CMM has three steps: 1) Construct a dependencies graph from the sequential algorithm in a restricted form called Linear Recursive Algorithm; 2) From the dependencies graph form a signal flow graph (SFG); and 3) From the SFG design a systolic array processor. This paper focused exclusively on the design of the systolic array and no experimental results were presented.

In [9], the authors presented data parallel algorithms for computing 3rd and 4th order moments on the MasPar-1 SIMD parallel system. Their program handles input sequences of length up to $2^{10}$. Their algorithm can be thought of as a mesh algorithm. No time complexity analyses were given in the paper and the algorithm was very specific for the MasPar-1 machine.

In [10] also, the authors considered the parallel computation of the bispectrum. They implemented the direct and indirect methods using two different parallel programming techniques: semiautomatic and fully automatic using the Power C Analyzer. For the semi-automatic technique, they achieved a speedup of 10.84 with 12 processors (for the direct method) and a speedup of 8.7 on 10 processors (for the indirect method). Using the fully parallel technique, they got a speedup of 10.07 with 11 processors (for the direct method) and a speedup of 7.67 with 12 processors (for the indirect method). The machine used was the Silicon Graphics Power
Challenge MIMD Machine HOTBLACK. This paper also falls under the category of developing a program for a specific machine.

\section{A BETTER SEQUENTIAL ALGORITHM FOR THE DIRECT METHOD}

In this section we show how to improve the run time of the direct method from $O\left(M n M_{3}^{2}\right)$ to $O(M n)$. The new algorithm is based on an efficient way of computing window sums that we describe next.

\subsection{Computing Window Sums}

The case of 1D data: Let $X=k_{1}, k_{2}, \ldots, k_{n}$ be any sequence of real numbers and let $w$ be a window size. The problem is to compute $s_{i}=\sum_{j=1}^{w} k_{i+j-1}$, for $1 \leq i \leq(n-w+1)$.

A straightforward algorithm for this problem will take $O(n w)$ time. We can improve the run time to $O(n)$ using the fact that successive window sums have significant overlaps. Specifically, $s_{i+1}=s_{i}-k_{i}+k_{i+j}$, for $1 \leq i \leq(n-w)$. This means that $s_{i+1}$ can be obtained from $s_{i}$ in $O(1)$ time. Therefore, if we compute the window sums in this order: $s_{1}, s_{2}, \ldots, s_{n-w+1}$, then we can compute all of them in $O(n)$ time.

The case of 2D data: The above idea can be extended to $2 \mathrm{D}$ data as well. Let $A=\left(a_{i, j}\right)$ be an $n \times n$ matrix and let $w$ be a window size. Consider the problem of computing $s_{i, j}=\sum_{u=1}^{w} \sum_{v=1}^{w} a_{i+u-1, j+v-1}$, for $1 \leq i \leq(n-w+1)$ and $1 \leq j \leq(n-w+1)$.

A trivial algorithm for solving the above problem will take $O\left(n^{2} w^{2}\right)$ time. We can improve this run time to $O\left(n^{2}\right)$ as follows.

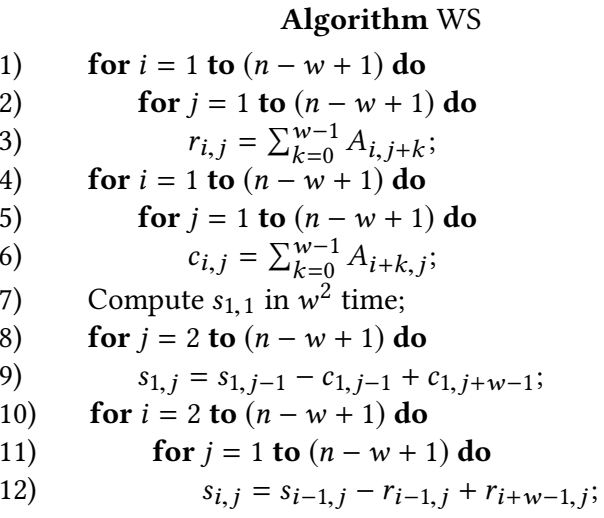

Time Complexity Analysis: For a given value of $i$, the for loop of line 2 corresponds to computing the window sums in row $i$ and hence the for loop of line 2 can be completed in $O(n)$ time. In turn, the for loop of line 1 takes $O\left(n^{2}\right)$ time. Similarly, the for loop of line 4 corresponds to computing window sums for each column of $A$. The total time needed is $O\left(n^{2}\right)$. Step 7 takes $w^{2}$ time. The for loop of line 8 takes $O(n)$ time. Line 12 takes $O(1)$ time per operation and hence the for loop of line 10 takes $O\left(n^{2}\right)$ time. In all, the total run time of the above algorithm is $O\left(n^{2}\right)$.

\subsection{Direct Method for the Bispectrum}

We employ the above window sums algorithms in the smoothing step (5) of the direct method and we can get the following theorem.

THEOREM 3.1. We compute the bispectrum of any input of size $n$ using the direct method in $O(M n)$ time, $M$ being the partition size. $\square$ 


\section{PARALLEL MODELS OF COMPUTING}

In this section we describe the parallel models of computing that we employ in this paper, namely, the PRAM and the mesh. A Parallel Random Access Machine (PRAM) is a collection of RAMs working in synchrony where communication takes place with the help of a common block of shared memory [6,7]. For instance if processor $i$ wants to communicate with processor $j$, it can do so by writing a message in memory cell $j$ which can then be read by processor $j$. More than one processor may want to either write into or read from the same cell at the same time. Depending on how these conflicts are handled, a PRAM can further be classified into three categories: Exclusive Read and Exclusive Write (EREW) PRAM, Concurrent Read and Exclusive Write (CREW) PRAM, and Concurrent Read and Concurrent Write (CRCW) PRAM. In the case of a CRCW PRAM, it is essential to have a mechanism for handling write conflicts, since the processors trying to write at the same time in the same cell can possibly have different data to write and we should determine which data gets written. This is not a problem in the case of concurrent reads since the data read by different processors will be the same. In a Common-CRCW PRAM, concurrent writes are permissible only if the processors trying to access the same cell have the same data to write. In an Arbitrary-CRCW PRAM, if more than one processor tries to write in the same cell at the same time, an arbitrary one of them succeeds. In a Priority-CRCW PRAM, write conflicts are resolved using processors' pre-assigned priorities.

An $n \times n$ mesh can be represented as a directed $n \times n$ grid-graph whose nodes correspond to processing elements and whose edges correspond to bidirectional communication links [6, 11]. If two processors are connected by an edge, they can communicate in a unit step. Otherwise, they communicate by sending a message along a connecting path.

The work done by a parallel algorithm that uses $P$ processors and runs in time $T$ is defined as the product $P \times T$.

\subsection{Some Fundamental Parallel Algorithms}

In this section we describe some basic algorithms that will be employed in our parallel algorithms. These algorithms can be found in relevant texts (such as $[6,7,11]$ ).

Let $\oplus$ be any associative unit-time computable binary operator defined in some domain $\Sigma$. Given a sequence of $n$ elements $k_{1}, k_{2}, \ldots, k_{n}$ from $\Sigma$, the problem of prefix computation is to compute $k_{1}, k_{1} \oplus k_{2}, \ldots, k_{1} \oplus k_{2} \oplus \cdots \oplus k_{n}$.

Lemma 4.1. Prefix computation on a sequence of n elements can be performed in $O(\log n)$ time using $\frac{n}{\log n}$ EREW PRAM processors.

Lemma 4.2. Prefix computation on a sequence of $n$ elements can be performed in $O(n)$ time on a linear array of size $n$.

Lemma 4.3. Prefix computation on a sequence of $n^{2}$ elements can be performed in $O(n)$ time on a $n \times n$ mesh.

Lemma 4.4. The slow-down lemma: Any PRAM algorithm that runs in time $T$ using $P$ processors can be simulated on a $P^{\prime}$-processor machine in time $O\left(\frac{P T}{P^{\prime}}\right)$, for any $P^{\prime} \leq P$.

\subsection{Window Sums on PRAM}

We now show how to implement the direct method on an EREW PRAM optimally. First we consider the problem of computing window sums in $1 \mathrm{D}$ and $2 \mathrm{D}$.

The case of 1D data in parallel: Let $X=k_{1}, k_{2}, \ldots, k_{n}$ be any sequence of real numbers and let $w$ be a window size. The problem is to compute $s_{i}=\sum_{j=1}^{w} k_{i+j-1}$, for $1 \leq i \leq(n-w+1)$.

A straightforward PRAM algorithm for this problem could use $(n-w+1)$ processors. Each processor can in parallel compute one window sum in $O(w)$ time. The work done will be $O(n w)$. We can improve these bounds using the prefix computation.

1) Perform a prefix sums computation on $k_{1}, k_{2}, \ldots, k_{n}$. Let the results be $q_{1}, q_{2}, \ldots, q_{n}$; Let $q_{0}=0$;

2) for $i=1$ to $(n-w+1)$ in parallel do

3) $s_{i}=q_{i+w-1}-q_{i-1}$;

Time Complexity Analysis: Step 1 can be done using $\frac{n}{\log n}$ EREW PRAM processors in $O(\log n)$ time (c.f. Lemma 4.1). The for loop of line 2 can be performed in $O(1)$ time using $n$ EREW PRAM processors. Using the slow-down lemma (c.f. Lemma 4.4), Step 2 can also be completed in $O(\log n)$ time using $\frac{n}{\log n}$ processors. Thus we can conclude as follows.

Lemma 4.5. The window sums computation problem on any input sequence of length $n$ can be solved in $O(\log n)$ time using $\frac{n}{\log n}$ EREW PRAM processors. $\square$

The case of $2 \mathrm{D}$ data in parallel: Let $A=\left(a_{i, j}\right)$ be an $n \times n$ matrix and let $w$ be a window size. We are interested in computing $s_{i, j}=$ $\sum_{u=1}^{w} \sum_{v=1}^{w} a_{i+u-1, j+v-1}$, for $1 \leq i \leq(n-w+1)$ and $1 \leq j \leq$ $(n-w+1)$.

A trivial algorithm for solving the above problem will do $O\left(n^{2} w^{2}\right)$ work. We can improve this work to $O\left(n^{2}\right)$ as follows. In this algorithm, $t_{i, 0}=0$, for $1 \leq i \leq(n-w+1)$.

\section{Algorithm WS_PRAM}

\section{for $j=1$ to $n$ in parallel do}

Compute window sums in column $j$; Specifically, let $c_{i, j}=\sum_{k=0}^{w-1} a_{i+k, j}$; for $1 \leq i \leq(n-w+1)$;

3) for $i=1$ to $(n-w+1)$ in parallel do

4) Perform a prefix sums computation on $c_{i, 1}, c_{i, 2}, \ldots, c_{i, n}$ Let the results be $t_{i, 1}, t_{i, 2}, \ldots, t_{i, n}$;

5) for $i=2$ to $(n-w+1)$ in parallel do

6) for $j=1$ to $(n-w+1)$ in parallel do

7) $s_{i, j}=t_{i, j+w-1}-t_{i, j-1}$;

Time Complexity Analysis: In line 1, for a specific value of $j$, window sums can be computed in $O(\log n)$ time using $\frac{n}{\log n}$ EREW PRAM processors (c.f. Lemma 4.5). Thus the for loop of line 1 can be completed in $O(\log n)$ time given $\frac{n^{2}}{\log n}$ EREW PRAM processors.

In line 3 , for any given value of $i$, prefix sums computation can be performed in $O(\log n)$ time using $\frac{n}{\log n}$ EREW PRAM processors (c.f. Lemma 4.1). As a result, the for loop of line 3 takes $O(\log n)$ time given $\frac{n^{2}}{\log n}$ EREW PRAM processors. 
Line 7 can be performed (for a given $i$ and $j$ ) in $O(1)$ time using one processor. Therefore, the for loop of line 5 can be performed in $O(1)$ time given $(n-w+1)^{2}$ EREW PRAM processors. Using the slow-down lemma (c.f. Lemma 4.4), the for loop of line 5 can also be completed in $O(\log n)$ time using $\frac{n^{2}}{\log n}$ processors.

Jointly considered, the above algorithm runs in a total of $O(\log n)$ time using $\frac{n^{2}}{\log n}$ EREW PRAM processors. Clearly, this algorithm is asymptotically work-optimal. We arrive at the following theorem:

THEOREM 4.6. The window sums computation problem can be solved in $O(\log n)$ time using $\frac{n^{2}}{\log n}$ EREW PRAM processors. $\square$

\subsection{Direct Method for Bispectrum on PRAM}

In this section we present a PRAM algorithm for bispectrum computation based on the direct method. There are 6 steps in the algorithm (c.f. Algorithm DBS). We discuss how to parallelize each step. Let $X(1), X(2), \ldots, X(n)$ be the input sequence.

\section{Algorithm DBS_PRAM}

1) Partition the data into $K$ parts with $M$ samples in each and this does not cost any time since the input will be given in the common memory. Let the parts be $X^{i}, 1 \leq i \leq K$.

2) Finding the mean of $X^{i}$ can be done in $O(\log M)$ time using $\frac{M}{\log M}$ processors, for a specific $i$. Thus the mean of all the parts can be found in $O(\log M)$ time using $\frac{n}{\log M}$ processors. Using the slow down lemma (c.f. Lemma 4.4), Step 2 can be performed in $O(\log n)$ time using $\frac{n}{\log n}$ EREW PRAM processors.

3) Compute the Discrete Fourier Transform (DFT) $F^{x^{i}}(k)$ for each part: $F^{x^{i}}(k)=\sum_{u=0}^{M-1} X^{i}(u) e^{-j \frac{2 \pi}{M} u k}$, for $k=0,1, \ldots, M-$ $1 ; i=1,2, \ldots, K$. For each part, the time taken is $O(\log M)$ using $M$ processors (see e.g., $[6,7])$. Therefore, the DFT of all the parts can be computed in $O(\log M)$ time using $n$ processors.

4) We estimate the third order spectrum of each part. Specifically, we have to compute $C_{3}^{F^{x^{i}}}\left(k_{1}, k_{2}\right)$, for $1 \leq i \leq K$ and $0 \leq k_{2} \leq$ $k_{1}, k_{1}+k_{2}<M / 2$. This can be done in $O(1)$ time using $O(n M)$ processors. Equivalently, Step 4 can also be done in $O(\log n)$ time using $\frac{n M}{\log n}$ EREW PRAM processors (using the slow down lemma).

5) Smoothing operation is conducted. The value of the bispectrum at any point is computed as an average over a surrounding window of size $M_{3} \times M_{3}$. This Step can be performed using the Algorithm WS_PRAM (c.f. Theorem 4.6). For each part, this Step can be completed in $O(\log M)$ time using $\frac{M^{2}}{\log M}$ processors. For all the $K$ parts together, Step 5 takes $O(\log M)$ time using $\frac{n M}{\log M}$ processors. Using the slow down lemma, Step 5 can be completed in $O(\log n)$ time employing $\frac{n M}{\log n}$ processors.

6) The bispectrum is computed as the average over all parts. In particular, we have to compute

$$
\hat{C}_{3}^{x}\left(\omega_{1}, \omega_{2}\right)=\frac{1}{K} \sum_{i=1}^{K} \tilde{C}_{3}^{x^{i}}\left(\omega_{1}, \omega_{2}\right), \omega_{1}=\frac{2 \pi}{M} k_{1} \text { and } \omega_{2}=\frac{2 \pi}{M} k_{2} .
$$

For a given $\omega_{1}$ and $\omega_{2}, \hat{C}_{3}^{x}\left(\omega_{1}, \omega_{2}\right)$ can be computed using a prefix sums computation on $K$ elements and hence can be done in $O(\log K)$ time using $\frac{K}{\log K}$ processors. Thus Step 6 can be completed in $O(\log K)$ time using $\frac{M^{2} K}{\log K}=\frac{n M}{\log K}$ processors. The slow down lemma implies that Step 6 can also done in $O(\log n)$ time using $\frac{n M}{\log n}$ processors.

In summary, we get the following theorem.

THEOREM 4.7. We can compute the bispectrum on any sequence of length $n$ in $O(\log n)$ time using $\frac{n M}{\log n}$ EREW PRAM processors, where $M$ is the size used to partition the input sequence. $\square$

\section{MEMORY EFFICIENT ALGORITHMS}

Note that the size of the bispectrum on any input of length $n$ is $O\left(M^{2}\right)$, where $M$ is the size used to partition the input. If $M$ is large, this amount of memory may not be available in a typical machine (such as a desktop). In this section we show how to reduce the memory requirement. Specifically, we prove that the memory requirement can be reduced to $O\left(M M_{3}\right)$ where $M_{3}$ is the window size used in the smoothing step. Sequentially, the time complexity remains the same as before (i.e., $O(n M)$ ). On the EREW PRAM, the work done remains the same. However, the run time increases to $O(M \log n)$ (using $\frac{n}{\log n}$ processors). From a practical perspective, we may not have a finer parallelism than this. I.e., the number of processors available may not be more than $\frac{n}{\log n}$. Thus our algorithm can be thought of as optimal from a perspective of not only the work done, but also the amount of run time achievable.

\subsection{D Window Sums}

We start with the problem of window sums computation in 2D. Consider the problem of computing window sums in an $n \times n$ matrix, $w$ being the window size. The idea is to compute one row at a time. At any given time we keep $w$ rows of $A$ in memory.

\section{Algorithm WS_LowMemory}

1) To begin with we keep the first $w$ rows of $A$. Let $c_{i, j}=$ $\sum_{k=0}^{w-1} a_{i+k, j}$, for $1 \leq i \leq(n-w+1)$. We start by computing $c_{1, j}$, for $1 \leq j \leq n$. This can be done in $O(\log w)$ time using $\frac{n w}{\log w}$ EREW PRAM processors. An application of the slow down lemma implies that this step can also be completed in $O(w \log n)$ time using $\frac{n}{\log n}$ processors.

2) Followed by this we perform a perfix sums computation on $c_{1,1}, c_{1,2}, \ldots, c_{1, n}$. Let $t_{1,1}, t_{1,2}, \ldots, t_{1, n}$ be the result. This takes $O(\log n)$ time using $\frac{n}{\log n}$ processors.

3) Next, we compute $s_{1, j}=t_{1, j+w-1}-t_{1, j-1}$, for $1 \leq j \leq(n-$ $w+1)$, where $t_{1,0}=0$. This can be done in $O(\log n)$ time using $\frac{n}{\log n}$ processors.

This completes the window sums computations for the first row. Now we bring row $(w+1)$ of $A$ from the disk (or any secondary storage) and proceed to compute the window sums corresponding to the second row of $A$.

4) In general before starting the computation of window sums corresponding to row $i$, rows $i-1, i, i+1, \ldots,(i+w-2)$ of $A$ will be in memory (for $2 \leq i \leq(n-w+1)$ ). We bring row $i+w-1$ from the disk. After computing the window sums for row $i$, we get rid of row $i-1$ from memory. Window sums for row $i$ are computed as follows:
a) for $j=1$ to $n$ do
b) $\quad c_{i, j}=c_{i-1, j}-a_{i-1, j}+a_{i+w-1, j}$;
c) Perform a prefix sums computation on $c_{i, 1}, c_{i, 2}, \ldots, c_{i, n}$. 


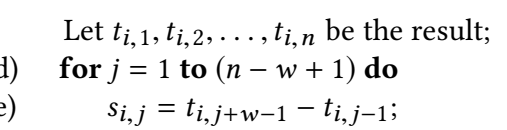

The for loop of line a) can be done in $O(\log n)$ time using $\frac{n}{\log n}$ processors. Line c) takes $O(\log n)$ time with the use of $\frac{n}{\log n}$ processors. The for loop of line d) can also be performed in $O(\log n)$ time using $\frac{n}{\log n}$ EREW PRAM processors.

To summarize, the memory we utilize is $(w+1) n=O(n w)$. The total time taken in line 1 to line 3 is $O(w \log n)$. In line 4 , the time spent for each of the rows $2,3, \ldots$, is $O(\log n)$. Thus the total time spent is $O(n \log n)$. The number of processors used is $\frac{n}{\log n}$ throughout. Thus the total work done is $O\left(n^{2}\right)$ which is clearly optimal asymptotically. We get the following theorem:

Theorem 5.1. We can solve the window sums problem on any $n \times n$ matrix in $O(n \log n)$ time using $\frac{n}{\log n}$ EREW PRAM processors using only $O(n w)$ memory, $w$ being the window size. $\square$

\subsection{Direct Method with $O\left(M M_{3}\right)$ Memory}

In this section we discuss how to reduce the memory used in the direct method. Specifically, we will use only $O\left(M M_{3}\right)$ memory, where $M$ is the size of each part and $M_{3}$ is the window size in smoothing.

\section{Algorithm DBS_LowMemory (Fast)}

1) Partitioning the input $X$ into $K$ parts does not cost any time.

2) We do the computations one part at a time. For each part we spend $O(\log M)$ time using $\frac{M}{\log M}$ EREW PRAM processors. The memory used is $O(M)$ and the time taken is $O\left(\frac{n}{M} \log M\right)$. Since we have $O\left(M M_{3}\right)$ space, we can work on $M_{3}$ parts in parallel. In this case, the time will reduce to $O\left(\frac{n}{M M_{3}} \log M\right)$ and the processor bound will be $\frac{M M_{3}}{\log M}$. If we use $\frac{M}{\log n}$ processors, the time will be $\mathrm{O}\left(\frac{n}{M} \log n\right)$.

3) The DFT of all parts can be computed in $O\left(\frac{n}{M M_{3}} \log M\right)$ time using $M M_{3}$ processors and $O\left(M M_{3}\right)$ memory. If we have $\frac{M}{\log n}$ processors this will take $O\left(\frac{n}{M} \log ^{2} n\right)$ time.

4) This step can be done in $O\left(\frac{n}{M_{3}}\right)$ time using $M M_{3}$ EREW PRAM processors. If we have $\frac{M}{\log n}$ processors, Step 4 can be completed in $O(n \log n)$ time.

5) Smoothing operation is conducted. The value of the bispectrum at any point is computed as an average over a surrounding window of size $M_{3} \times M_{3}$. This Step can be performed using the window sums Algorithm WS_LowMemory (c.f. Theorem 5.1). For each part, this Step can be completed in $O(M \log M)$ time using $\frac{M}{\log M}$ processors and $O\left(M M_{3}\right)$ memory. For all the $K$ parts together, Step 5 takes $O(n \log M)$ time using $\frac{M}{\log M}$ processors. Using the slow down lemma, Step 5 can be completed in $O(n \log n)$ time employing $\frac{M}{\log n}$ processors.

6) The bispectrum is computed as the average over all parts. In particular, we have to compute

$$
\hat{C}_{3}^{x}\left(\omega_{1}, \omega_{2}\right)=\frac{1}{K} \sum_{i=1}^{K} \tilde{C}_{3}^{x^{i}}\left(\omega_{1}, \omega_{2}\right), \omega_{1}=\frac{2 \pi}{M} k_{1} \text { and } \omega_{2}=\frac{2 \pi}{M} k_{2} .
$$

For a given $\omega_{1}$ and $\omega_{2}, \hat{C}_{3}^{x}\left(\omega_{1}, \omega_{2}\right)$ can be computed using a prefix sums computation on $K$ elements and hence can be done in $O(\log K)$ time using $\frac{K}{\log K}$ processors. Thus Step 6 can be completed in $O\left(\frac{M M_{3}}{\log n}\right)$ time using $\frac{n \log n}{M_{3}}$ processors. The slow down lemma implies that Step 6 can also done in $O(n \log n)$ time using $\frac{M}{\log n}$ processors.

THEOREM 5.2. Bispectrum computation on any given sequence of length $n$ can be computed in $O(n \log n)$ time using $\frac{M}{\log n}$ EREW PRAM processors and $O\left(M M_{3}\right)$ memory, where $M$ is the size of each part and $M_{3}$ is the window size of smoothing. $\square$

\subsection{Algorithms Using Even Less Memory}

In this section we present an algorithm that uses only $O\left(w^{2}\right)$ memory. The work done is still $O\left(n^{2}\right)$. Let $A$ be the input $n \times n$ matrix. We partition the matrix into submatrices of size $w \times w$ each. Label these matrices as $A_{i, j}$ where $1 \leq i, j \leq \frac{n}{w}$ and the submatrix $A_{1,1}$ refers to the $w \times w$ submatrix of $A$ in the top left corner. We compute the window sums corresponding to each submatrix at a time.

Consider any submatrix $A_{i, j}$. We want to compute the window sums corresponding to each entry in $A_{i, j}$. If $a_{k, l}$ is any element in the matrix $A$, then the window sum corresponding to this element is nothing but the sum of all the elements in the $w \times w$ submatrix whose top left corner is $a_{k, l}$.

In order to compute the window sums corresponding to $A_{i, j}$ we bring the following four $w \times w$ submatrices from the disk: $A_{i, j}, A_{i, j+1}, A_{i+1, j}$ and $A_{i+1, j+1}$. Note that the window sums corresponding to $A_{i, j}$ depend only on these four submatrices. We apply Lemma 4.6 on these four submatrices with $n=4 w$. For each $A_{i, j}$, the computation takes $O(\log w)$ time using $\frac{w^{2}}{\log w}$ processors. Using the slow-down lemma, this can also be done in $O(\log n)$ time and $\frac{w^{2}}{\log n}$ processors.

Summing all the submatrices, the time spent is $O\left(\log n \times \frac{n^{2}}{w^{2}}\right)$. Processor and memory bounds are $\frac{w^{2}}{\log n}$ and $O\left(w^{2}\right)$, respectively. We get the following theorem:

THEOREM 5.3. Window sums on any $n \times n$ data can be computed in $O\left(\frac{n^{2}}{w^{2}} \log n\right)$ time using $\frac{w^{2}}{\log n}$ EREW PRAM processors and $O\left(w^{2}\right)$ memory, $w$ being the window size. $\square$

Along the same lines, we can also prove the following theorem:

THeOREM 5.4. Window sums on any $n \times n$ data can be computed in $O\left(n^{2} \log n\right)$ time using $\frac{w}{\log n}$ EREW PRAM processors and $O(w)$ memory, $w$ being the window size. $\square$

Note that the work done in the above algorithm is $O\left(n^{2} w\right)$ and hence the algorithm is not work optimal. However, the memory used is very small. Theorems $4.6,5.1,5.3$, and 5.4 consider memories of different specific sizes. Theorems 4.6 and 5.3 can be used to develop work optimal algorithms when the memory available is $m$ for any $w^{2} \leq m \leq n^{2}$. 


\section{MESH ALGORITHMS}

In this section we consider solving the problem of computing HOS spectra on an $n \times n$ mesh.

\subsection{Computing DFT}

Let $X=x_{1}, x_{2}, \ldots, x_{n}$ be any given sequence of data. The DFT of $X$ can be thought of as the values of the polynomial $f(x)=$ $x_{1}+x_{2} x+x_{3} x^{2}+\ldots x_{n} x^{n-1}$ at the $n$th roots of unity (see e.g., [6]). The $n$th roots of unity are $1, \omega, \omega^{2}, \ldots, \omega^{n-1}$ where $\omega=e^{2 \pi i / n}$ is the primitive $n$th root of unity. Let $g_{i}=f\left(\omega^{i}\right)$, for $0 \leq i \leq(n-1)$.

We show below that we can compute the DFT of any sequence of length $n$ on an $n \times n$ mesh in $O(n)$ time. Let the nodes of the mesh be numbered $(i, j)$ where $1 \leq i, j \leq n$. Assume that the input is given in the first row of the mesh one element per node. Specifically, $x_{i}$ is input in node $(1, i), 1 \leq i \leq n$. Row $i$ will compute $g_{i}$ in $O(n)$ time, $1 \leq i \leq n$.

\section{Algorithm DFT_Mesh}

1) Node $(i, j)$ computes $\omega^{(i-1)(j-1)}$, for $1 \leq i, j \leq n$. This is done using the repeated squaring method in $O(\log n)$ time;

2) Broadcast $x_{i}$ along column $i$, for $1 \leq i \leq n$;

3) Node $(i, j)$ computes $d_{i, j}=x_{i} \omega^{(i-1)(j-1)}$, for $1 \leq i, j \leq n$;

4) Row $i$ computes $g_{i}=\sum_{j=1}^{n} d_{i, j}$ using Lemma 4.2;

5) At the end of Step $4, g_{i}$ is available at node $(i+1, n), 0 \leq i \leq$ $(n-1)$. Route these values to row 1 . In particular, route $g_{i}$ to node $(1, i+1)$, for $0 \leq i \leq(n-1)$. This routing is done using the shortest greedy paths.

Analysis: Line 1 takes $O(\log n)$ time. Line 2 takes $(n-1)$ steps. Line 3 takes one unit of time. Sum computation in line 4 can be done using a prefix computation in $O(n)$ time (c.f. Lemma 4.2). Line 5 also can be done in $O(n)$ time since the maximum distance traveled by any element is $n$ and there are no collisions in the routing process. Thus we end up with the following Theorem:

THEOREM 6.1. DFT computation on any sequence of length $n$ can be done in $O(n)$ time on an $n \times n$ mesh.

\subsection{Direct Method on an $n \times n$ Mesh}

Now we present a mesh algorithm for the direct computation of bispectrum. We will consider Steps 1 through 6 of Algorithm DBS. The input is given in row 1 of the mesh.

\section{Algorithm DBS_Mesh}

1) Step 1 does not involve any computation;

2) In Step 2, we have to compute the average value of each part. We can do this using Lemma 4.2. The idea is to perform this computation in parallel on every subarray of size $M$ in row 1 . Specifically, the subarray corresponding to the first part will be the nodes $(1,1),(1,2), \ldots,(1, M)$; The subarray corresponding to part 2 will be the nodes $(1, M+1),(1, M+2), \ldots,(1,2 M)$ and so on;

3) Compute the DFT of the input using the Algorithm DFT_Mesh (c.f. Theorem 6.1);

4) In Step 4, the third order spectrum computation happens within each part. Therefore, these computations happen within subarrays of size $M \times M$. Consider any node in any of these subarrays. Without loss of generality consider the top leftmost such subarray. Let $(i, j)$ be any node in this subarray. The third order spectrum value at this node is computed as $g_{i-1}+g_{j-1}+g_{(i-1)+(j-1)}$. In order to do this we perform a series of routings. Specifically, we broadcast $g_{i-1}$ along row $i, 1 \leq i \leq n$; broadcast $g_{j-1}$ along column $j, 1 \leq j \leq n$; and broadcast $g_{(i-1)+(j-1)}$ along the diagonal $(i+j)$, $1 \leq i, j \leq n$. After this broadcasting is completed, each node has all the elements it needs to compute a spectrum value;

5) Step 5 can be done using the strategy of Algorithm WS. In particular, we compute window sums along each column in parallel. Followed by this we perform prefix sums computations along each row $i, 1 \leq i \leq\left(n-M_{3}+1\right)$;

6) Finally, Step 6 can be done by first routing the values from the $K$ parts to the first part; followed by this, each node in the top left $M \times M$ subarray computes the needed averages.

Analysis: Step 2 takes $O(M)=O(n)$ time. Step 3 takes $O(n)$ time (c.f. Theorem 6.1). In Step 4, the routing takes $O(n)$ time and the spectrum computation takes $O(1)$ time. Steps 5 and 6 also take $O(n)$ time each.

The above algorithm and analysis yield the following theorem:

THEOREM 6.2. Bispectrum computation of a sequence of length $n$ can be performed on an $n \times n$ mesh in $O(n)$ time.

\section{GENERAL POLYSPECTRAL ESTIMATION}

We have thus far focused on the computation of the bispectrum. The same sequential and parallel techniques can also be extended to higher order spectra. Consider, for example, the fourth order spectrum (also called trispectrum). The computation will be very similar to that in Algorithm DBS. In Step 1 we partition the input into $K$ parts. Step 2 is the computation of the averages of each part and subtracting the mean. Step 3 computes the DFT. In Step 4, fourth order spectra are estimated in each part. In particular, we compute: $C_{4}^{x^{i}}\left(k_{1}, k_{2}, k_{3}\right)=F^{x^{i}}\left(k_{1}\right) F^{x^{i}}\left(k_{2}\right) F^{x^{i}}\left(k_{3}\right) F^{x^{i}}\left(k_{1}+k_{2}+k_{3}\right)$ for $1 \leq k_{1}, k_{2}, k_{3} \leq M$. This takes time $O\left(n M^{2}\right)$. Step 5 involves smoothing over a subcube of size $\left(M_{3} \times M_{3} \times M_{3}\right)$. A straightforward algorithm take $O\left(n M^{2} M_{3}^{3}\right)$ time to do this smoothing operation. We can apply the overlap technique employed in Algorithm WS to complete this step in $O\left(n M^{2}\right)$ time. Step 6 in involves averaging over the $K$ parts and will take $O\left(n M^{2}\right)$ time. This will yield the following theorem:

THEOREM 7.1. We can compute fourth order spectrum on any input of size $n$ in $O\left(n M^{2}\right)$ time where $M$ is the size of each part.

All of our parallel techniques (for the PRAM and the mesh) and memory reduction techniques also apply for the fourth order spectrum, trispectrum. Due to space constraints, we only show their experiment results in Section 8. In fact they apply for $k$ th order spectrum for any $k \geq 3$. For instance, for the $k$ th order spectrum, the following theorem holds for sequential computation:

THEOREM 7.2. On any input of size $n$, we can compute $k$ th order spectrum in $O\left(n M^{k-2}\right)$ time, for any $k \geq 3$ where $M$ is the size of each part in the input.

\section{EXPERIMENTAL RESULTS}

In this section we show extensive experiment results on evaluating the performance of our proposed approaches. 


\subsection{Test Platform}

All the experiments have been performed on Dell Precision Workstation T7910, which is equipped with Intel(R) Xeon(R) CPU E5-2667 v3 @ 3.20GHz, with 16 cores (hyperthreading to 32 threads), 256 GB of main memory and 4 TB of HDD disk. All the algorithms have been implemented using $\mathrm{C}++$ and the standard GCC compiler. The parallel version is implemented using OpenMP, which is a shared memory multi-threads library integrated in GCC.

\subsection{Datasets and Experimental Settings}

Though our proposed algorithms should be data independent, to offer an unbiased evaluation, we have generated different types of time series for our experiments. Specifically, we have followed the guidelines given in [4] to generate ten different types of time series data, with different lengths $(n)$. The generating functions are:

- Linear autoregressive processes:

$$
x_{t}=\alpha x_{t-1}+\epsilon_{t}, \alpha= \pm 0.9,0.4
$$

- Bilinear processes [17]:

$$
\begin{aligned}
& x_{t}=0.4 x_{t-1}+\alpha x_{t-1} \epsilon_{t-1}+\epsilon_{t}, \alpha=0.4,0.6 \\
& x_{t}=-0.2 x_{t-1}+0.4 x_{t-2}+0.6 x_{t-1} \epsilon_{t-1}+0.7 x_{t-2} \epsilon_{t-1}+\epsilon_{t}
\end{aligned}
$$

- Self-exciting threshold autoregressive processes [20]:

$$
\begin{aligned}
& x_{t}=\left\{\begin{array}{c}
1.227+1.0516 x_{t-1}-0.8901 x_{t-2}-0.2149 x_{t-3} \\
+\epsilon_{t}^{\prime}, \text { if } x_{t-1} \leq 0.5 \\
1.6734-0.8295 x_{t-1}+0.1309 x_{t-2}-0.0276 x_{t-3} \\
+\epsilon_{t}^{\prime}, \text { otherwise }
\end{array}\right. \\
& x_{t}=\left\{\begin{array}{c}
0.15+0.85 x_{t-1}+0.22 x_{t-2}-0.7 x_{t-3}+\epsilon_{t}^{\prime}, \\
\text { if } x_{t-1} \leq 3.05 \\
0.3-0.8 x_{t-1}+0.2 x_{t-2}+0.7 x_{t-3}+\epsilon_{t}^{\prime}, \\
\text { otherwise }
\end{array}\right.
\end{aligned}
$$

- EXPAR process [8]:

$$
x_{t}=0.5 x_{t-1}+1.5 x_{t-1} e^{-0.5 x_{t-1}^{2}}+\epsilon_{t}
$$

- POLYAR process [4]:

$x_{t}=0.3452 x_{t-1}+0.1204 x_{t-1}^{2}-0.0994 x_{t-2}+0.1162 x_{t-1} x_{t-2}+\epsilon_{t}$ where $\epsilon_{t} \sim N(0,1)$ and $\epsilon_{t}^{\prime} \sim N\left(0,0.003^{2}\right)$ are Gaussian white noise. In the experiments, we have randomly picked sequences from these 10 types, truncated them to a certain length $n$, and used them as the input data for our algorithms. Without losing generality, we use a value of $K=1$ throughout, where the input sequence has only one part of size $n$. We use conventional linear smoothing window with window width $M_{3}=\left\|n^{0.625}\right\|$ (closest odd integer) [1] For bispectrum experiments, we have used the following values: $n=2^{7}, 2^{8}, \ldots, 2^{16}$; and for trispectrum, due to a dimension increase, we have employed the following values: $n=2^{7}$ to $2^{12}$.

We have implemented algorithms for spectral computation up to the fourth order, i.e., for bispectrum and trispectrum computations. Higher order approaches are similar and it is easy to extend our techniques to solve them. For both bispectrum and trispectrum, we have compared 5 different approaches: Naive approach with $O\left(n^{2} M_{3}^{2}\right)$ run time, denoted as Naive; Our sequential algorithm that takes $O\left(n^{2}\right)$ time (c.f. Theorem 3.1) - Call this algorithm as WS; Our fastest algorithm in Section 5.2 that does $O\left(n^{2}\right)$ work and uses $O\left(n M_{3}\right)$ space - Call this algorithm Fast; The memory-efficient algorithm introduced in Section 5.3 which uses only $O\left(M_{3}^{2}\right)$ memory - Call this algorithm Efficient; Parallel approach (denoted as
Parallel) using different number of cores $2,4,8,16$. Also we use the prefixes Bi- or Tri- to refer to the bispectrum and trispectrum implementations of these algorithms, respectively. We have evaluated our proposed approaches from both the running time and the maximum memory usage perspectives.

\subsection{Sequential Algorithm Comparisons}

We have set a run time threshold of 10 hours and a memory threshold of 100 GB. Any algorithm exceeding one or both of these thresholds was forced to stop. For large datasets such as those with $n=2^{15}, 2^{16}, 2^{17}$, some of the algorithms exceeded these thresholds. In Figure 2, we show the running time of different approaches for bispectrum and trispectrum, respectively.

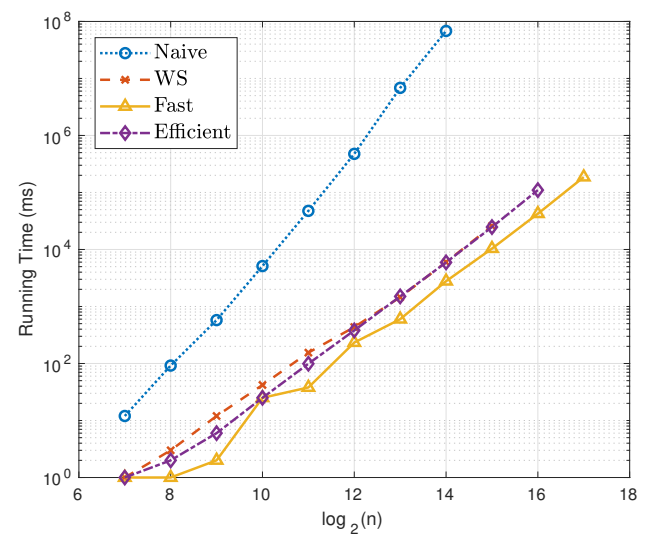

(a) Bispectrum Runtime

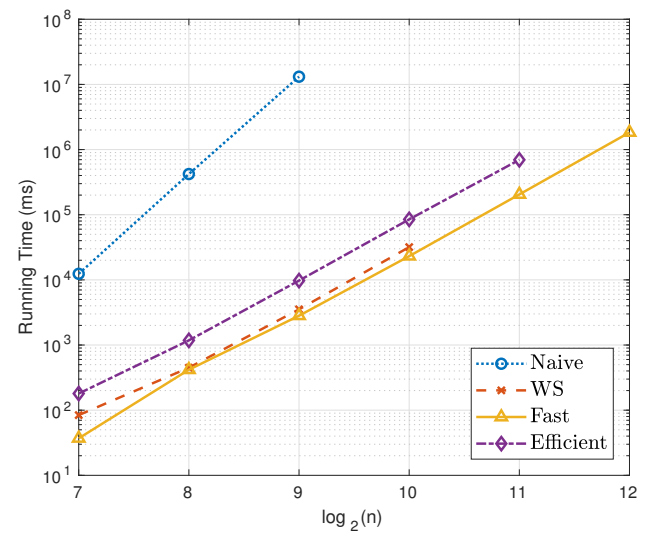

(b) Trispectrum Runtime

\section{Figure 2: Runtime Comparison for Different Values of $n$}

Note that this is a semilog plot on the Y-axis. Thus the parallel curves show orders of magnitude difference. From this figure we can clearly see that compared with the naive algorithm, all of our algorithms offer much better run times. It is important to notice that the Fast approach outperforms all the others in terms of speed in both bispectrum and trispectrum computations. Although WS is a simpler method, it takes more time than Fast in practice which could be due to cache misses. 
We also provide the maximum memory usage comparison among the algorithms in Table 1 . We can see that the memory cost in our experiments is consistent with our theoretical analysis. As it is shown, the Efficient approach is extremely memory efficient. For instance, for the time sequence with a length of $n=2^{16}$, it only requires less than $100 \mathrm{MB}$ of memory. In contrast, even the second memory efficient approach Fast uses around $1 \mathrm{~GB}$, and the others occupy more than $30 \mathrm{~GB}$. Efficient would take a longer time than Fast, which demonstrates the trade-off between space and time.

Table 1: Memory Usage (in MB) Comparison

\begin{tabular}{c|cccc}
\hline $\mathrm{n}$ & Naive & WS & Fast & Efficient \\
\hline \multicolumn{5}{|c}{ Bispectrum } \\
\hline $2^{9}$ & 10.0 & 10.0 & 2.8 & 2.6 \\
$2^{10}$ & 33.1 & 32.9 & 3.6 & 2.6 \\
$2^{11}$ & 126.7 & 126.7 & 6.3 & 2.8 \\
$2^{12}$ & 504.8 & 504.7 & 14.5 & 5.2 \\
$2^{13}$ & 2030.7 & 2030.5 & 38.6 & 9 \\
$2^{14}$ & 8176.3 & 8176.3 & 113.8 & 17.6 \\
$2^{15}$ & NA & 32890 & 344.3 & 37.9 \\
$2^{16}$ & NA & NA & 1055.8 & 86.3 \\
\hline \hline & \multicolumn{4}{c}{ Trispectrum } \\
\hline $2^{7}$ & 56.8 & 56.7 & 8.4 & 3.6 \\
$2^{8}$ & 448 & 448 & 38.2 & 7.3 \\
$2^{9}$ & 3682.7 & 3682.7 & 215.8 & 19.3 \\
$2^{10}$ & NA & 30224.3 & 1297.7 & 64.6 \\
$2^{11}$ & NA & NA & 7869.3 & 228.4 \\
\hline
\end{tabular}

Additionally, we have also cross-compared running times of bispectrum computation with the HOSA Toolbox in MATLAB [18] for single thread. Table 2 shows how fast our Fast implementation becomes when series lengths increase. We have computed bispectrum with linear smoothing window for both of these implementations. Our package can be a meaningful alternative for HOS estimation.

Table 2: Runtime (in sec) Comparison with HOSA Toolbox

\begin{tabular}{cccc}
\hline $\mathbf{n}$ & window size & Fast & HOSA Toolbox \\
\hline 128 & $21 \times 21$ & 0.0012 & 0.01 \\
256 & $33 \times 33$ & 0.0048 & 0.042 \\
512 & $49 \times 49$ & 0.0114 & 0.22 \\
1024 & $77 \times 77$ & 0.0316 & 1.755 \\
2048 & $117 \times 117$ & 0.1258 & 23.342 \\
4096 & $181 \times 181$ & 0.448 & 329.961 \\
8192 & $279 \times 279$ & 1.7514 & 3102.4 \\
\hline
\end{tabular}

\subsection{Multi-Core Parallel Approach Evaluation}

In this section we evaluate our proposed parallel algorithm. Due to the fact that the Efficient approach has a significant advantage in memory, we have implemented the parallel version of Efficient to offer a fast and memory efficient approach in high order spectra computations. We have tested the Parallel algorithms using different number of cores $2,4,8,16$ and $n$ from $2^{7}$ to $2^{16}$.

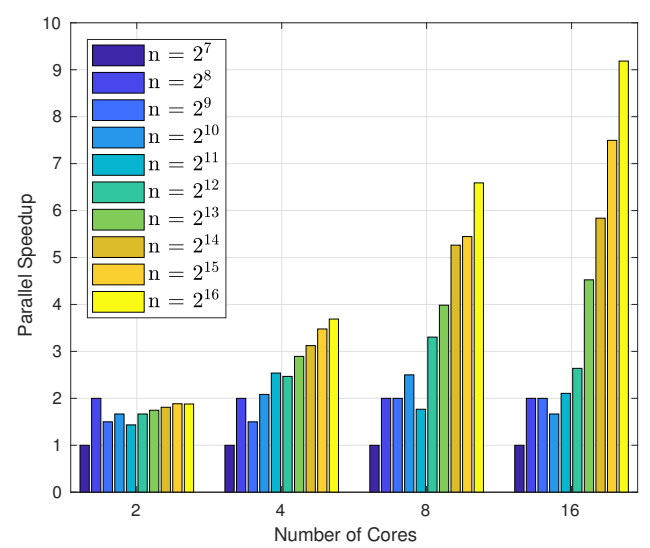

(a) Bispectrum Speedup

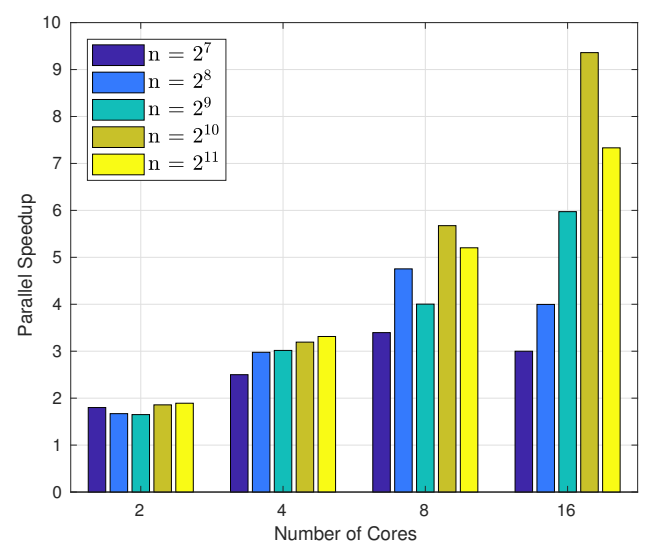

(b) Trispectrum Speedup

Figure 3: Speedups Using Different Numbers of Cores

In Figure 3, we plot the parallel speedup against number of cores. As we can see, from 2 cores to 16 cores, the speedup is nearly doubled if the number of cores is doubled, meaning that an optimal speedup is achieved. An interesting fact is that for short sequences (small $n$ values), the gain of multi-cores is not as significant as for larger $n$ values. This is due to the overhead in multi-core implementations, such as processor scheduling and communication. For small datasets, the overhead of work scheduling becomes dominant. On the contrary, the computation time is still dominant for long time series. This makes our parallel algorithm especially useful for HOS estimation of large datasets.

For memory usage, we provide the maximum memory cost for our parallel implementation in Figure 4. As expected, the memory cost for the parallel implementation is linearly dependent on the number of cores. This is due to the fact that each processor is working on its own smoothing window independently from each other. This means that our algorithm is also space optimal and only uses a small amount of memory for very large $n$ values, e.g., 250 MB for $n=2^{15}$ when 32 cores (hyperthreading) are utilized. 


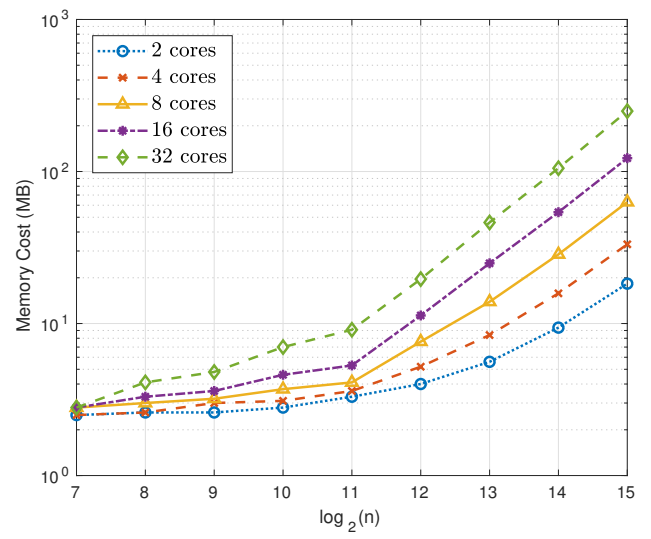

(a) Bispectrum Memory Usage

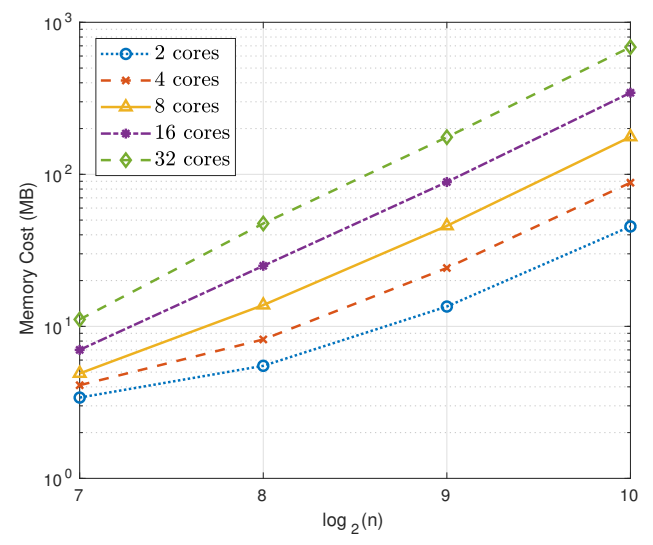

(b) Trispectrum Memory Usage

Figure 4: Memory Usage by Different Numbers of Cores

\subsection{Summary of Experiments}

In this section, we have evaluated four approaches, Naive, WS, Fast, and Efficient, respectively, as well as the parallel algorithm. Both bispectrum and trispectrum implementations have been tested on different lengths of time series data. The following critical observations are made from these experiments.

- All of our proposed algorithms outperform the naive algorithm by orders of magnitude, in terms of both run time and memory cost. Please note that the naive algorithm is the best one published in the literature. Fast is $25 \times 10^{3}$ times faster when $n=2^{15}$, and Efficient uses less than $1 / 200$ th of the memory used by the Naive algorithm.

- Even though WS is simpler, Fast still runs the fastest. It could be due to the cache misses and memory accessing time costs, as WS occupies a significantly larger memory.

- Fast and Efficient display a memory-time trade-off. Fast has a better balance and Efficient is frugal in memory usage.

- Parallel is a fast and memory saving algorithm, suitable for problems with very large $n$ values. A linear speedup can be achieved by Parallel on larger datasets. The memory cost for Parallel is also linearly dependent on the number of cores.

\section{CONCLUSIONS}

In this paper we offer efficient sequential and parallel algorithms for computing HOS. The work done by our algorithms is asymptotically better than any known algorithms. We propose efficient sequential HOS estimation algorithms with optimal run time and parallel algorithm with linear speedup. Another crucial problem in computing HOS is in the need for large memories. To address this problem, we offer memory efficient algorithms for both sequetial and parallel cases. Experimental results reveal that our algorithms are highly competitive and especially suitable for long sequences.

\section{ACKNOWLEDGMENTS}

This work has been supported in part by the following NSF grants: 1447711, 1514357, 1743418, and 1843025.

\section{REFERENCES}

[1] H Akaike, T Ozaki, M Ishiguro, Y Ogata, G Kitagawa, YH Tamura, E Arahata, K Katsura, and Y Tamura. 1984. Time Series Analysis and Control Program Package, TIMSAC-84. The Institute of Statistical Mathematics, Tokyo (1984).

[2] Elie Bou Assi, Laura Gagliano, Sandy Rihana, Dang K Nguyen, and Mohamad Sawan. 2018. Bispectrum Features and Multilayer Perceptron Classifier to Enhance Seizure Prediction. Scientific reports 8, 1 (2018), 15491.

[3] Azadeh Moradinezhad Dizgah, Hayden Lee, Julian B Muñoz, and Cora Dvorkin. 2018. Galaxy bispectrum from massive spinning particles. Fournal of Cosmology and Astroparticle Physics 2018, 05 (2018), 013.

[4] Jane L Harvill, Nalini Ravishanker, and Bonnie K Ray. 2013. Bispectral-based methods for clustering time series. Computational Statistics \& Data Analysis 64 (2013), 113-131.

[5] Ganesh Hegde and R Chris Bowen. 2017. Machine-learned approximations to density functional theory hamiltonians. Scientific reports 7 (2017), 42669.

[6] Ellis Horowitz, Sartaj Sahni, and Sanguthevar Rajasekaran. 1997. Computer algorithms $\mathrm{C}++$ : $\mathrm{C}++$ and pseudocode versions. Macmillan.

[7] Joseph JáJá. 1992. An introduction to parallel algorithms. Vol. 17. Addison-Wesley Reading.

[8] David Alan Jones. 1978. Nonlinear autoregressive processes. Proceedings of the Royal Society of London. A. Mathematical and Physical Sciences 360, 1700 (1978), 71-95.

[9] John N Kalamatianos and Elias S Manolakos. 1995. Parallel computation of higher order moments on the MasPar-1 machine. In 1995 International Conference on Acoustics, Speech, and Signal Processing, Vol. 3. IEEE, 1832-1835.

[10] Khoa N Le, Gregory K Egan, and Kishor P Dabke. 1999. Parallel computation of the bispectrum. In ISSPA'99. Proceedings of the Fifth International Symposium on Signal Processing and its Applications, Vol. 1. IEEE, 251-254.

[11] F Thomson Leighton. 2014. Introduction to parallel algorithms and architectures: Arrays- trees-hypercubes. Elsevier.

[12] Elias S. Manolakos, Haris M. Stellakis, and Dana H. Brooks. 1991. Parallel processing for biomedical signal processing. Computer 24, 3 (1991), 33-43.

[13] VV Naumenko, GA Proskura, AV Totsky, and Karen O Egiazarian. 2018. Bispectrum-based ultra wide band communication system. Telecommunications and Radio Engineering 77, 10 (2018).

[14] Jonathan M Nichols, Colin C Olson, Joseph V Michalowicz, and Frank Bucholtz. 2009. The bispectrum and bicoherence for quadratically nonlinear systems subject to non-Gaussian inputs. IEEE Transactions on signal processing 57, 10 (2009), 3879-3890.

[15] Chrysostomos L Nikias and Jerry M Mendel. 1993. Signal processing with higherorder spectra. IEEE Signal processing magazine 10, 3 (1993), 10-37.

[16] Athina P. Petropulu. 2000. Higher-Order Spectral Analysis. CRC Press.

[17] T Subba Rao and MM Gabr. 2012. An introduction to bispectral analysis and bilinear time series models. Vol. 24. Springer Science \& Business Media.

[18] Ananthram Swami, Jerry Mendel, and Chrysostomos Nikias. 2001. Higher-order spectral analysis toolbox: for use with MATLAB, The MathWorks and United Signals \& Systems, Inc. Retrieved Apr, 2018 from https://www.mathworks.com/ matlabcentral/fileexchange/3013-hosa-higher-order-spectral-analysis-toolbox

[19] Yumeng Tao, Xiaogang Gao, Alexander Ihler, Soroosh Sorooshian, and Kuolin Hsu. 2017. Precipitation identification with bispectral satellite information using deep learning approaches. fournal of Hydrometeorology 18, 5 (2017), 1271-1283.

[20] Howell Tong and Keng S Lim. 2009. Threshold autoregression, limit cycles and cyclical data. In Exploration Of A Nonlinear World: An Appreciation of Howell Tong's Contributions to Statistics. World Scientific, 9-56.

[21] Gaetano Valenza, Luca Citi, Antonio Lanatá, Enzo Pasquale Scilingo, and Riccardo Barbieri. 2014. Revealing real-time emotional responses: a personalized assessment based on heartbeat dynamics. Scientific reports 4 (2014), 4998. 